\title{
Desmin common mutation is associated with multi-systemic disease manifestations and depletion of mitochondria and mitochondrial DNA
}

\author{
Elizabeth M. McCormick ${ }^{1}$, Lawrence Kenyon ${ }^{2}$ and Marni J. Falk ${ }^{1,3 *}$ \\ 'Division of Human Genetics, Department of Pediatrics, The Children's Hospital of Philadelphia, Philadelphia, PA, USA, \\ ${ }^{2}$ Department of Pathology, Thomas Jefferson University Hospital, Philadelphia, PA, USA, ${ }^{3}$ Department of Pediatrics, \\ University of Pennsylvania Perelman School of Medicine, Philadelphia, PA, USA
}

OPEN ACCESS

Edited by:

Tiziana Lodi,

University of Parma, Italy

Reviewed by:

Michela Rugolo,

University of Bologna, Italy

Rita Barresi,

National Health Service, UK

*Correspondence:

Marni J. Falk,

The Children's Hospital of Philadelphia and University of Pennsylvania

Perelman School of Medicine, ARC 1002C, 3615 Civic Center BIvd,

Philadelphia, PA 19104, USA

falkm@email.chop.edu

Specialty section: This article was submitted to

Genetic Disorders,

a section of the journal

Frontiers in Genetics

Received: 30 January 2015 Accepted: 20 May 2015

Published: 05 June 2015

Citation:

McCormick EM, Kenyon L and Falk MJ (2015) Desmin common mutation

is associated with multi-systemic

disease manifestations and depletion of mitochondria and mitochondrial

DNA. Front. Genet. 6:199.

doi: 10.3389/fgene.2015.00199
Desmin $(D E S)$ is a major muscle scaffolding protein that also functions to anchor mitochondria. Pathogenic DES mutations, however, have not previously been recognized as a cause of multi-systemic mitochondrial disease. Here, we describe a 45-year-old man who presented to The Children's Hospital of Philadelphia Mitochondrial-Genetics Diagnostic Clinic for evaluation of progressive cardiac, neuromuscular, gastrointestinal, and mood disorders. Muscle biopsy at age 45 was remarkable for cytoplasmic bodies, as well as ragged red fibers and SDH positive/COX negative fibers that were suggestive of a mitochondrial myopathy. Muscle also showed significant reductions in mitochondrial content ( $16 \%$ of control mean for citrate synthase activity) and mitochondrial DNA (35\% of control mean). His family history was significant for cardiac conduction defects and myopathy in multiple maternal relatives. Multiple single gene and panel-based sequencing studies were unrevealing. Whole exome sequencing identified a known pathogenic p.S13F mutation in DES that had previously been associated with desmin-related myopathy. Desmin-related myopathy is an autosomal dominant disorder characterized by right ventricular hypertrophic cardiomyopathy, myopathy, and arrhythmias. However, neuropathy, gastrointestinal dysfunction, and depletion of both mitochondria and mitochondrial DNA have not previously been widely recognized in this disorder. Recognition that mitochondrial dysfunction occurs in desmin-related myopathy clarifies the basis for the multi-systemic manifestations, as are typical of primary mitochondrial disorders. Understanding the mitochondrial pathophysiology of desmin-related myopathy highlights the possibility of new therapies for this otherwise untreatable and often fatal class of disease. We postulate that drug treatments aimed at improving mitochondrial biogenesis or reducing oxidative stress may be effective therapies to ameliorate the effects of desmin-related disease.

Keywords: desmin-related myopathy, DES, mitochondrial disease, whole exome sequencing, mitochondrial DNA depletion 


\section{Introduction}

A 45-year-old Caucasian man presented to The Children's Hospital of Philadelphia Mitochondrial-Genetics Diagnostic Clinic for evaluation of suspected mitochondrial disease based on arrhythmia, cardiomyopathy, progressive lower extremity myopathy, and peripheral neuropathy. His cardiac problems presented as episodic dizziness and congenital heart block that were noted in his late $20 \mathrm{~s}$, with a pacemaker placed at 30 years of age. Over the next decade, he developed intermittent atrial flutter, right hypertrophic cardiomyopathy, and congestive heart failure. Myopathic symptoms began in his mid-late $30 \mathrm{~s}$ with leg weakness that progressed following pneumonia at 38 years of age. By age 45 , he was no longer able to run and had significant difficulty ambulating. Electromyogram performed at that time revealed a distal myopathy with myotonic discharges and mild length-dependent neuropathy. A detailed review of systems was significant for lifelong short stature, progressive photophobia, hoarse voice, gastroesophageal reflux, swallowing dysfunction, progressive truncal obesity, progressive bloating, anger, and intermittent depression. Review of prior laboratory screening studies were significant for an elevated GGT, elevated indirect bilirubin, and elevated creatine kinase (315 U/L). Prior genetic testing only included $D M 1$ and $D M 2$ expansion analyses, which were normal.

His family history was significant for multiple individuals with cardiac and/or neuromuscular disease (Figure 1).

The proband's physical examination at age 45 was significant for short stature, distant heart sounds, abdominal obesity, small hands, and cold and purplish-colored feet. He was unable to get onto the exam table without assistance, but could lie back and sit up with minimal assistance. He had profound thigh weakness and was unable to lift either thigh even slightly without resistance. He had normal 5/5 strength of the upper extremities, $4 / 5$ strength in the fingers, and 3-4/5 strength in the lower legs and feet with significant calf muscle wasting. He had $1+$ biceps and patellar deep tendon reflexes, but ankle reflexes could not be elicited. He had a slapping gait, was unable to perform toe or heel walking, and had difficulty performing tandem gait. He was unable to stoop at the knees and recover. He reported sharp and dull sensation on his feet and legs.

Clinical follow-up at age 46 was notable for interval development of progressive leg muscle weakness and tingling, especially of the calves, progressive atrophy of arm and leg muscles, increased leg swelling, and a constant cold sensation of his legs. Physical examination revealed cervical kyphosis and $2+$ pitting edema of the legs with swollen ankles obscuring all landmarks. He no longer could sit up with minimal assistance but instead needed to roll onto his side to get from a supine to a sitting position. Upper extremity strength was stable. He reported an inability to feel the floor with his feet and now had only minimal control of foot and ankle movements. Patellar and ankle DTRs could not be elicited.

Metabolic laboratory studies performed at age 45 were normal, including a comprehensive chemistry panel, complete blood count, lipoprotein profile, thyroid function screen, plasma and urine amino acid quantitative analyses, pyruvate, lactate,

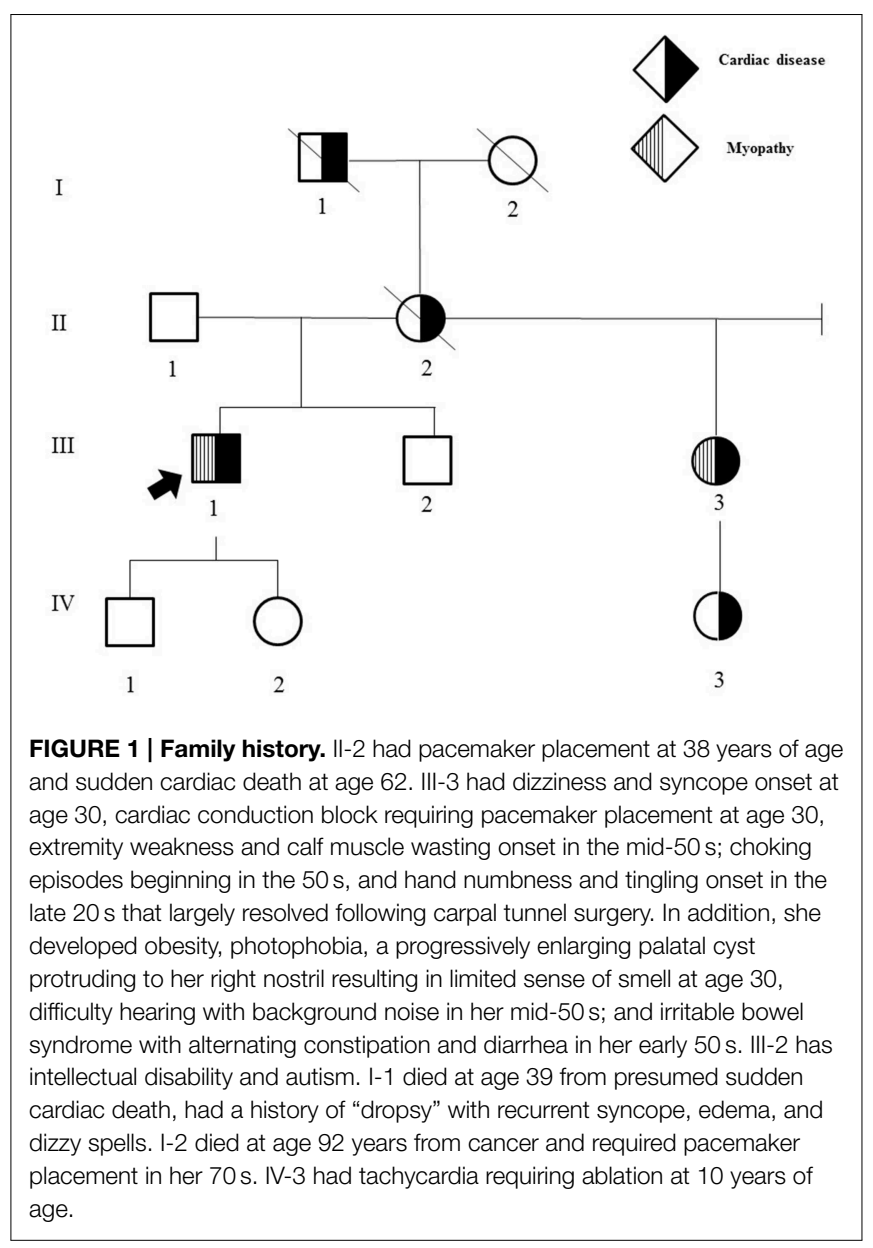

ammonia, carnitine analysis, acylcarnitine profile, urinalysis, and urine organic acid analysis, with the only exception being a mildly elevated creatine kinase activity ( $258 \mathrm{U} / \mathrm{L}$, normal 55-170 U/L).

Left quadriceps muscle biopsy was performed at age 45 , which revealed generalized muscle fiber atrophy with fatty-fibrous tissue infiltration. Ragged red fibers and SDH positive/COX negative fibers consistent with a mitochondrial myopathy were identified (Figure 2). No ultrastructural mitochondrial morphologic abnormalities were evident by electron microscopy although two cytoplasmic bodies were present.

Muscle mitochondrial DNA content by quantitative PCR analysis revealed mitochondrial DNA depletion, at 35\% of the mean value of age- and tissue-matched controls. Per Baylor Medical Genetics Clinical Diagnostic Laboratory standard techniques, mitochondrial and nuclear DNA contents are determined by real-time quantitative PCR using specific primers for the mitochondrial tRNALeu(UUR) gene and the nuclear $ß$ 2-microglobulin (B2M) gene (Bai et al., 2004; Bai and Wong, 2005; Dimmock et al., 2008, 2010; Wong et al., 2008; Venegas et al., 2011). The mitochondrial DNA copy number is calculated from the difference in threshold cycle numbers of mitochondrial DNA and nuclear DNA. Control samples were obtained from over 300 individuals suspected of having mitochondrial disease but mutations in genes known to cause mitochondrial DNA depletion were not identified. 


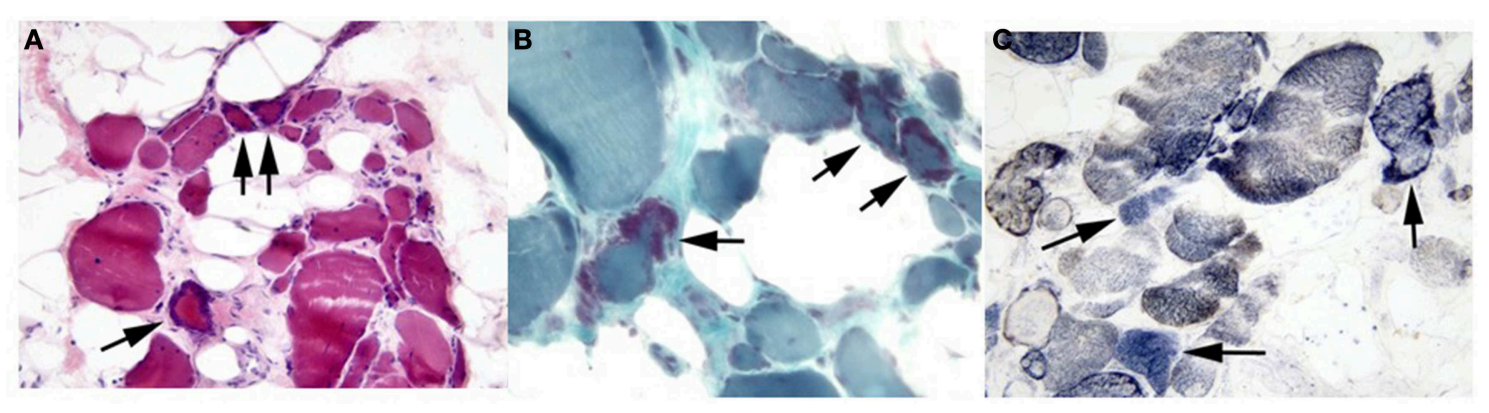

FIGURE 2 | Proband's skeletal muscle cryostat sections. Muscle sections stained with (A) H\&E or (B) modified Gomori trichrome stain demonstrate marked variability in fiber size, with fiber atrophy and fatty infiltration. Ragged red fibers are marked by arrows in both panels. (C) $\mathrm{SDH} / \mathrm{COX}$ staining demonstrated several fibers (arrow) with SDH activity (blue) that lack COX activity (brown). Original magnifications: (A), 200X; (B,C), 400X.
Muscle mitochondrial DNA sequencing by next-generation sequencing did not detect large deletions or known deleterious point mutations.

Muscle mitochondrial respiratory chain enzyme activity analyses revealed significant deficiency of overall mitochondrial content as evidenced by citrate synthase activity reduced to $16 \%$ of the control mean; relative deficiency of several electron transport chain enzyme activities was also present that largely normalized (complex II+III) or improved to $>30 \%$ of control (complex II, complex IV) when corrected for the low citrate synthase activity (Table 1). This reduction after correction for citrate synthase may suggest that, in addition to reduced mitochondrial mass, the energetic efficiency may be compromised. Per Baylor Medical Genetics Clinical Diagnostic Laboratory standard technique, the electron transport chain enzymes were assayed at $30^{\circ} \mathrm{C}$ using a temperature-controlled spectrophotometer. The activities of complex I, complex II, complex I+III, complex II+III, and complex IV were each measured in duplicate using different electron acceptors/donors. The increase or decrease of cytochrome $c$ at $550 \mathrm{~nm}$ was measured for complex I+III, II+III, or complex IV. The activity of complex I was measured by following the oxidation of NADH at $340 \mathrm{~nm}$. For complex II, the reduction of 2,6dichloraindophenol (DCIP) at $600 \mathrm{~nm}$ was measured. Enzyme activities were normalized against citrate synthase activity (Ye and Hoppel, 2013; Vigelsø et al., 2014) as citrate synthase was greater than one standard deviation above or below control mean (Trounce et al., 1996; Walker et al., 1996; Bernier et al., 2002; Gellerich et al., 2004; Enns et al., 2005; Kirby et al., 2007). The 95\% confidence interval is used as control, with several hundred individuals included in each control range. Additionally, a concurrent mouse muscle wild type control is analyzed with each enzymologic assay for quality control.

Genetic testing was performed in sequential fashion. At age 45, blood-based mitochondrial DNA sequencing by next-generation sequencing analysis and frataxin gene expansion analysis were normal. Following muscle biopsy studies, LMNA sequencing was recommended and was normal, as was sequencing, deletion, and duplication analysis of 101 nuclear genes known to be associated with mitochondrial disease. Whole exome sequencing (WES) on a clinical diagnostic basis at Baylor Medical Genetics Laboratory was then recommended, although no parental samples were available for analysis. WES identified a known mutation in the DES gene, namely c.38C $>\mathrm{T} ; \mathrm{p} . \mathrm{S} 13 \mathrm{~F}$, that was previously associated with desmin-related myopathy. Specifically, the p.S13F mutation had previously been reported as a diseasecausing founder mutation in Dutch patients reported to have skeletal muscle weakness and cardiac involvement (Bergman et al., 2007; van Spaendonck-Zwarts et al., 2012). Targeted p.S13F mutation analysis in his symptomatic, maternal half-sister was positive and negative in his niece with a history of tachycardia requiring ablation at 10 years of age. The family is considering targeted mutation analysis in proband's full brother and children.

\section{Background}

Desmin-related myopathy caused by missense mutations in $D E S$ has been recognized to be a fully penetrant, autosomal dominant condition with variable manifestations that largely include myopathy, cardiomyopathy, and arrhythmias (Goldfarb et al., 1998; Dalakas et al., 2000; Bergman et al., 2007; van Spaendonck-Zwarts et al., 2012). Desmin expression is highest in heart, followed by muscle, and gastrointestinal tract ${ }^{1}$. However, there is also expression known in the peripheral nerve and reproductive organs including cervix, prostate, and uterus. The p.S13F mutation identified in the family described here is a previously identified, well-characterized pathogenic alteration and was first recognized as a disease-causing founder mutation in Dutch patients with varying degrees of skeletal muscle weakness and cardiac involvement (Dalakas et al., 2000). Prior phenotypic analysis of individuals carrying the p.S13F mutation in DES found that all individuals had cardiac conduction disease and/or cardiomyopathy while neuromuscular features were not present in everyone (van Spaendonck-Zwarts et al., 2012). Both the proband and his maternal half-sister in the present family had cardiac and neuromuscular involvement, both of which were more severe and progressed at an earlier age in the proband,

\footnotetext{
${ }^{1}$ GeneHub-GEPIS. Available online at: http://research-public.gene.com/cgibin/genentech/genehub-gepis/web_search-result.pl?id=GENE2507\&CHR=CHR2 $\&$ CHR_END $=219999704 \&$ CHR_START $=219991342 \& D E S C T=$ desmin $\& E N D=4$ 68412689\&GENE_NAME=DES\&ID=GENE2507\&SPECIES=HUMAN\&START $=$ 468404327\&STRAND $=1$ \&intype $=1$ \&xrefid=DES\&species $=$ human
} 
TABLE 1 | Muscle mitochondrial respiratory chain enzyme activity analyses.

\begin{tabular}{|c|c|c|c|c|c|}
\hline $\begin{array}{l}\text { Electron transport chain } \\
\text { activities }\end{array}$ & $\begin{array}{l}\text { ETC } \\
\text { complexes }\end{array}$ & $\begin{array}{l}\text { Percent of mean } \\
\text { (before correction for } \\
\text { decreased citrate } \\
\text { synthase) (\%) }\end{array}$ & $\begin{array}{l}\text { Percent of mean (after } \\
\text { correction for } \\
\text { decreased citrate } \\
\text { synthase) }(\%)\end{array}$ & $\begin{array}{c}\text { Patient activity } \\
\text { (nmol/min/mg protein) }\end{array}$ & $\begin{array}{l}\text { Control } \pm \text { standard } \\
\text { deviation }(\mathrm{nmol} / \mathrm{min} / \mathrm{mg} \\
\text { protein) }\end{array}$ \\
\hline $\begin{array}{l}\text { NADH:cytochrome c } \\
\text { reductase (rotenone } \\
\text { sensitive) }\end{array}$ & Complex I+III & 0 & 0 & 0 & $9.1 \pm 2.5$ \\
\hline Cytochrome c oxidase & Complex IV & 6 & 39 & 1.8 & $29.2 \pm 9.1$ \\
\hline Citrate synthase & & 16 & 100 & 44 & $280 \pm 95$ \\
\hline
\end{tabular}

as well as gastrointestinal dysfunction in both and progressive neuropathy and mood disorder in the proband. Our proband also had two cytoplasmic bodies identified on his muscle biopsy histology, which are not specific but can be seen in desminrelated myopathies. While the proband's niece with a history of tachycardia in childhood did not harbor the familial DES mutation, there are many recognized non-inherited causes of arrhythmia that could have been the source in this individual.

\section{Discussion}

While the cardiac and neuromuscular phenotype of desminrelated myopathy is well characterized, there is scarce reported literature on desmin-related myopathies being associated with mitochondrial dysfunction or disease. Desmin (DES) is a key scaffolding protein found in muscles that is known to function as a mitochondrial anchor (Stromer and Bendayan, 1988). Prior studies in desmin-null mice revealed subsarcolemmal accumulations of mitochondria in muscle and overall increased numbers of mitochondria, as well as impaired mitochondrial respiratory capacity (Milner et al., 2000). One previous case report in a man with a frameshift DES mutation (c.5141_5143insA; p.K239fsX242) revealed clustering of muscle mitochondria, normal respiratory chain enzymology in skeletal muscle homogenates, and decreased maximal rates of respiration and complex I in isolated saponin-permeabilized skeletal muscle fibers (Schroder et al., 2003). However, there was no report of decreased mitochondrial mass or mitochondrial DNA content either in that case or more generally in desmin-related myopathy. Additionally, two siblings with progressive muscle fatigue and weakness were found to be compound heterozygous for mutations in DES with carrier status confirmed in both asymptomatic parents. Muscle biopsy in one of these siblings revealed myopathic features, $5 \%$ COX deficient fibers on immunohistochemical analysis, and absent desmin staining. No mitochondrial DNA sequencing, mitochondrial DNA content analysis, nor enzymatic activity assays of mitochondrial respiratory chain function was reported.
Electron microscopy showed no structural abnormalities in the mitochondria although there was variability noted in their size and distribution (Henderson et al., 2013).

Additionally, while only muscle mitochondrial DNA content was assessed in this proband, it is not unreasonable to postulate that his multisystemic involvement can be due to muscle and/or peripheral nerve involvement. Indeed, neurogenic disease and/or polyneuropathy was reported on electromyogram analysis of at least three other individuals with DES mutations, including one confirmed to have the p.S13F mutation (Bergman et al., 2007).

\section{Concluding Remarks}

We report here a case of progressive cardiac and neuromuscular disease that was clinically suspected as a mitochondrial disorder based on his progressive, multi-systemic involvement including neurologic, gastrointestinal, and mood manifestations. Muscle biopsy in this case revealed ragged red fibers and $\mathrm{SDH}$ positive/COX negative fibers, findings consistent with classical primary mitochondrial disease, as well as pronounced depletion of mitochondrial DNA content and reduced mitochondrial mass, and complex I deficiency. Numerous single-gene and panelbased molecular analyses were unrevealing. Clinically-based whole exome sequencing revealed the underlying etiology in the proband and his similarly affected sister to be a previously reported pathogenic mutation in the DES gene. This case expands the phenotype of desmin-related myopathy to include clinical features in the nervous and gastroenterologic systems, as are typical of mitochondrial disease. Recognizing that significant mitochondrial depletion occurs in desmin-related myopathy is especially important to enable development of new therapeutic avenues for this progressive and often fatal disease, such as potentially through stimulation of mitochondrial biogenesis or reduction of oxidative stress that commonly occurs in mitochondrial disorders (Parikh et al., 2009). Finally, these data are suggestive that there may exist other neuromuscular diseases caused by mutations in structural proteins that interact directly with mitochondria. 


\section{References}

Bai, R. K., Perng, C. L., Hsu, C. H., and Wong, L. C. (2004). Quantitative PCR analysis of mitochondrial DNA content in patients with mitochondrial disease. Ann. N.Y. Acad. Sci. 1011, 304-309. doi: 10.1196/annals. 1293.029

Bai, R. K., and Wong, L. C. (2005). Simultaneous detection and quantification of mitochondrial DNA deletion(s), depletion, and over-replication in patients with mitochondrial disease. J. Mol. Diagn. 7, 613-622. doi: 10.1016/S15251578(10)60595-8

Bergman, J. E. H., Veenstra-Knol, H. E., van Essen, A. J., van Ravenswaaij, C. M. A., den Dunnen, W. F. A., van den Wijngaard, A., et al. (2007). Two related Dutch families with a clinically variable presentation of cardioskeletal myopathy caused by a novel S13F mutation in the desmin gene. Eur. J. Med. Genet. 50, 355-366. doi: 10.1016/j.ejmg.2007.06.003

Bernier, F. P., Boneh, A., Dennett, X., Chow, C. W., Cleary, M. A., and Thorburn, D. R. (2002). Diagnostic criteria for respiratory chain disorders in adults and children. Neurology 59, 1406-1411. doi: 10.1212/01.WNL.0000033795.17156.00

Dalakas, M. C., Park, K. Y., Semino-Mora, C., Lee, H. S., Sivakumar, K., and Goldfarb, L. G. (2000). Desmin myopathy, a skeletal myopathy with cardiomyopathy caused by mutations in the desmin gene. N. Engl. J. Med. 342, 770-780. doi: 10.1056/NEJM200003163421104

Dimmock, D. P., Zhang, Q., Dionisi-Vici, C., Carrozzo, R., Shieh, J., Tang, L. Y., et al. (2008). Clinical and molecular features of mitochondrial DNA depletion due to mutations in deoxyguanosine kinase. Hum. Mutat. 29, 330-331. doi: 10.1002/humu.9519

Dimmock, D., Tang, L. Y., Schmitt, E. S., and Wong, L. J. (2010). Quantitative evaluation of the mitochondrial DNA depletion syndrome. Clin. Chem. 56, 1119-1127. doi: 10.1373/clinchem.2009.141549

Enns, G. M., Hoppel, C. L., DeArmond, S. J., Schelley, S., Bass, N., Weisiger, K., et al. (2005). Relationship of primary mitochondrial respiratory chain dysfunction to fiber type abnormalities in skeletal muscle. Clin. Genet. 68, 337-348. doi: 10.1111/j.1399-0004.2005.00499.x

Gellerich, F. N., Mayr, J. A., Reuter, S., Sperl, W., and Zierz, S. (2004). The problem of interlab variation in methods for mitochondrial disease diagnosis: enzymatic measurement of respiratory chain complexes. Mitochondrion 4, 427-439. doi: 10.1016/j.mito.2004.07.007

Goldfarb, L. G., Park, K. Y., Cervenákova, L., Gorokhova, S., Lee, H. S., Vasconcelos, O., et al. (1998). Missense mutations in desmin associated with familial cardiac and skeletal myopathy. Nat. Genet. 19, 402-403. doi: $10.1038 / 1300$

Henderson, M., De Waele, L., Hudson, J., Eagle, M., Sewry, C., Marsh, J., et al. (2013). Recessive desmin-null muscular dystrophy with central nuclei and mitochondrial abnormalities. Acta Neuropathol. 125, 917-919. doi: 10.1007/s00401-013-1113-x

Kirby, D. M., Thorburn, D. R., Turnbull, D. M., and Taylor, R. W. (2007). Biochemical assays of respiratory chain complex activity. Methods Cell Biol. 80, 93-119. doi: 10.1016/s0091-679x(06)80004-x
Milner, D. J., Mavroidis, M., Weisleder, N., and Capetanaki, Y. (2000). Desmin cytoskeleton linked to muscle mitochondrial distribution and respiratory function. J. Cell Biol. 150, 1283-1298. doi: 10.1083/jcb.150.6.1283

Parikh, S., Saneto, R., Falk, M. J., Anselm, I., Cohen, B. H., and Haas, R. (2009). A modern approach to the treatment of mitochondrial disease. Curr. Treat. Options Neurol. 11, 414-430. doi: 10.1007/s11940-009-0046-0

Schroder, R., Goudeau, B., Casteras Simon, M., Fischer, D., Eggermann, T., Clemen, C. S., et al. (2003). On noxious desmin: functional effects of a novel heterozygous desmin insertion mutation on the extrasarcomeric desmin cytoskeleton and mitochondria. Hum. Mol. Genet. 12, 657-669. doi: 10.1093/hmg/ddg060

Stromer, M. H., and Bendayan, M. (1988). Arrangement of desmin intermediate filaments in smooth muscle cells as shown by highresolution immunocytochemistry. Cell Motil. Cytoskeleton 11, 117-125. doi: 10.1002/cm.970110205

Trounce, I. A., Kim, Y. L., Jun, A. S., and Wallace, D. C. (1996). Assessment of mitochondrial oxidative phosphorylation in patient muscle biopsies, lymphoblasts, and transmitochondrial cell lines. Meth. Enzymol. 264, 484-509. doi: 10.1016/s0076-6879(96)64044-0

van Spaendonck-Zwarts, K. Y., van der Kooi, A. J., van den Berg, M. P., Ippel, E. F., Boven, L. G., Yee, W. C., et al. (2012). Recurrent and founder mutations in the Netherlands: the cardiac phenotype of DES founder mutations p.S13F and p.N342D. Neth. Heart J. 20, 219-228. doi: 10.1007/s12471-011-0233-y

Venegas, V., Wang, J., Dimmock, D., and Wong, L. J. (2011). Real-time quantitative PCR analysis of mitochondrial DNA content. Curr. Protoc. Hum. Genet. Chapter 19:Unit 19.17. doi: 10.1002/0471142905.hg1907s68

Vigelsø, A., Andersen, N. B., and Dela, F. (2014). The relationship between skeletal muscle mitochondrial citrate synthase activity and whole body oxygen uptake adaptations in response to exercise training. Int. J. Physiol. Pathophysiol. Pharmacol. 6, 84-101.

Walker, U. A., Collins, S., and Byrne, E. (1996). Respiratory chain encephalomyopathies: a diagnostic classification. Eur. Neurol. 36, 260-267. doi: 10.1159/000117269

Wong, L. C., Naviaux, R. K., Brunetti-Pierri, N., Zhang, Q., Schmitt, E. S., Truong, C., et al. (2008). Molecular and clinical genetics of mitochondrial diseases due to POLG mutations. Hum. Mutat. 29, E150-E172. doi: 10.1002/humu.20824

Ye, F., and Hoppel, C. L. (2013). Measuring oxidative phosphorylation in human skin fibroblasts. Anal. Biochem. 437, 52-58. doi: 10.1016/j.ab.2013.02.010

Conflict of Interest Statement: The authors declare that the research was conducted in the absence of any commercial or financial relationships that could be construed as a potential conflict of interest.

Copyright (c) 2015 McCormick, Kenyon and Falk. This is an open-access article distributed under the terms of the Creative Commons Attribution License (CC BY). The use, distribution or reproduction in other forums is permitted, provided the original author(s) or licensor are credited and that the original publication in this journal is cited, in accordance with accepted academic practice. No use, distribution or reproduction is permitted which does not comply with these terms. 